\title{
Segmentation of Kidney and Renal Collecting System on 3D Computed Tomography Images
}

\author{
Bruno Oliveira ${ }^{1,2,3^{*}}$, Helena R. Torres ${ }^{1,2,3,{ }^{*},}$, Sandro Queirós ${ }^{1,2,3,4}$, Pedro Morais ${ }^{1,2,4,5}$, Jaime C. Fonseca ${ }^{3}$, Jan D’hooge ${ }^{4}$, \\ Nuno F. Rodrigues ${ }^{6}$, João L. Vilaça ${ }^{6}$ \\ ${ }^{1}$ Life and Health Sciences Research Institute (ICVS), School of Medicine, University of Minho, Braga, Portugal \\ ${ }^{2}$ ICVS/3B's - PT Government Associate Laboratory, Braga/Guimarães, Portugal \\ ${ }^{3}$ Algoritmi Center, School of Engineering, University of Minho, Guimarães, Portugal \\ ${ }^{4}$ Lab on Cardiovascular Imaging and Dynamics, Department of Cardiovascular Sciences, KULeuven - University of Leuven, \\ Leuven, Belgium \\ ${ }^{5}$ Instituto de Ciência e Inovação em Engenharia Mecânica e Engenharia Industrial, Faculdade de Engenharia, Universidade do \\ Porto, Portugal \\ ${ }^{6} 2 \mathrm{Ai}$ - Polytechnic Institute of Cávado and Ave, Barcelos, Portugal
}

\begin{abstract}
Surgical training for minimal invasive kidney interventions (MIKI) has huge importance within the urology field. Within this topic, simulate MIKI in a patient-specific virtual environment can be used for pre-operative planning using the real patient's anatomy, possibly resulting in a reduction of intra-operative medical complications. However, the validated VR simulators perform the training in a group of standard models and do not allow patient-specific training. For a patient-specific training, the standard simulator would need to be adapted using personalized models, which can be extracted from pre-operative images using segmentation strategies. To date, several methods have already been proposed to accurately segment the kidney in computed tomography (CT) images. However, most of these works focused on kidney segmentation only, neglecting the extraction of its internal compartments. In this work, we propose to adapt a coupled formulation of the B-Spline Explicit Active Surfaces (BEAS) framework to simultaneously segment the kidney and the renal collecting system (CS) from CT images. Moreover, from the difference of both kidney and CS segmentations, one is able to extract the renal parenchyma also. The segmentation process is guided by a new energy functional that combines both gradient and region-based energies. The method was evaluated in 10 kidneys from 5 CT datasets, with different image properties. Overall, the results demonstrate the accuracy of the proposed strategy, with a Dice overlap of $92.5 \%, 86.9 \%$ and $63.5 \%$, and a point-to-surface error around $1.6 \mathrm{~mm}, 1.9 \mathrm{~mm}$ and $4 \mathrm{~mm}$ for the kidney, renal parenchyma and CS, respectively.
\end{abstract}

Keywords- Computed Tomography, coupled B-Spline Explicit Active Surfaces, kidney segmentation, patient-specific virtual environment, renal collecting system segmentation;

\section{INTRODUCTION}

$\mathrm{M}$ INIMALLY invasive kidney interventions (MIKI) have become commonplace and have largely replaced traditional interventions (i.e. open surgery) [1,2]. Although providing considerable advantage to the patients, MIKI difficulty is relatively higher than traditional approaches

\footnotetext{
* First joint authorship
}

$[3,4]$. Recent clinical studies showed the added value of the VR systems to shorten the learning curve of several MIKI procedures when compared with the traditional mentor-based approach $[5,6]$. However, the validated VR simulators perform the training in a group of standard models and do not allow patient-specific training. In fact, a VR simulator that allows practicing in a patient-specific environment, prior to the intervention, can have a huge value for healthcare. Such tool can be useful not only for training but also to simulate any difficult step of a surgery, reducing, therefore, the probability of adverse events and the number of complications. For a patient-specific training, the standard simulator would need to be adapted using personalized models. These models can be extracted from pre-operative images, using segmentation strategies [7]. In fact, the necessity of a robust method to segment the kidney and its compartments could have hampered the development of such simulators.

To segment the kidney in CT images, (semi-)automatic segmentation strategies have been developed [8]. These strategies allow to reduce intra- and inter-observer variability, as well as minimize the time usually required for the traditional manual delineation. However, the task of segmenting the kidney in CT images is challenging owing to the low contrast between the kidney and its neighboring tissues.

Several approaches have been proposed in the literature for kidney segmentation, which include image-based methods [911], classification [12,13], atlas-based [14-16], or deformable model methods [17-24]. Deformable model approaches present advantages against other segmentation categories, namely by being robust to noise and by offering topological flexibility to the segmentation process. These approaches are based on the concept of active contours, which consist in a curve that is evolved through internal/external energies that represent the generic features used to separate the object of interest from the background. Smooth and gapless segmentations are typically extracted with this type of methods. Different works have used deformable models to segment the kidney. In [17], Qiao et al. proposed an active contour methodology where a gradient vector flow force is 

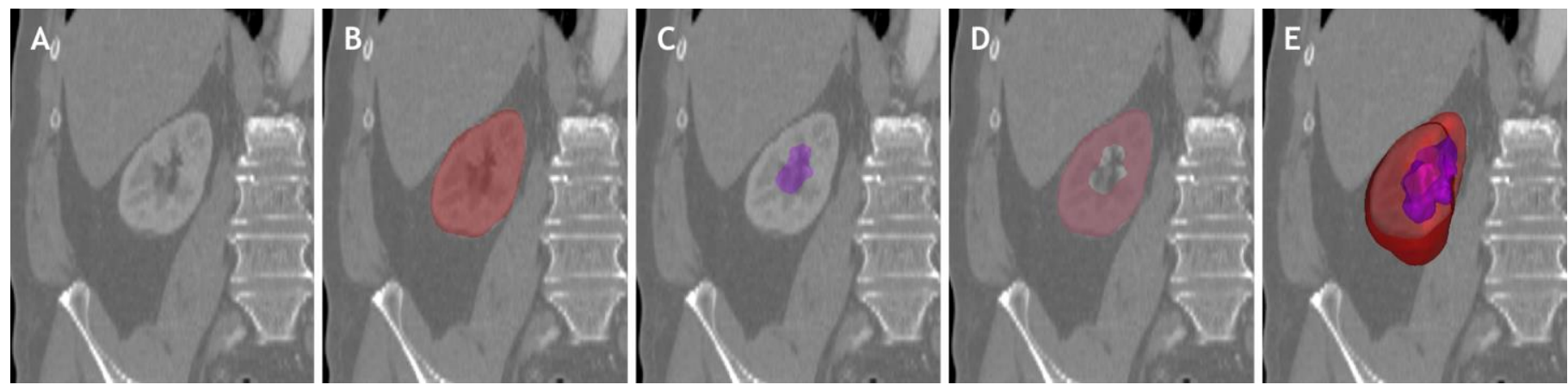

Figure 1 - CT image showing the different renal compartments. (A) CT image; (B) Kidney; (C) Renal collecting system; (D) Renal parenchyma; (E) 3D model of kidney and renal collecting system.

used to evolve the contour based on image characteristics. Another type of deformable model, namely template deformation, was used in $[18,19]$. In these works, the kidney's position is detected using contextual information, with an implicit template model being used to segment the kidney, driven by a kidney probability map constructed using random forests. A template deformation approach was also proposed in [20]. In this method, two types of deformation were explored, namely global and local deformations, which were constrained by local features. In [21,22], a level-set approach was used, which combined a statistical shape prior, visual descriptors, and homogeneity descriptors to evolve the contour. A level-set set method was also used by Hufnagel et al. [23]. In their work, the contour is evolved towards the kidney using image intensities and with constrains applied by a statistical shape model. Despite their relatively accurate results in segmenting the kidney, one common limitation across all these methods is their computational burden. To solve this problem, we previously proposed to use the B-spline Explicit Actives Surfaces (BEAS) framework to segment the kidney in [24], providing low computational time to the segmentation process.

Despite all the methods proposed in the literature for kidney segmentation in CT images, most are restricted to the extraction of one single solid structure. However, the kidney is internally divided in the renal parenchyma (which in its turn is divided in renal cortex and medulla) and in the renal collecting system (CS), which contains the renal pelvis and the sinus complex (see Figure 1). The segmentation of these different renal compartments in CT images has not been fully explored in the research field yet. However, the ability to distinguish both renal parenchyma and renal CS within the kidney can be useful for functional and morphological assessments of the kidney. In fact, the volume of the parenchyma can be helpful to identify certain pathological renal conditions, such as posterior urethral valves [25]. Similarly, the volume of the renal CS and its relation with the total volume of the kidney can help recognize renal diseases, such as hydronephrosis [25].

For the above reasons, the present work aims to develop a method to segment the different renal regions, namely the renal parenchyma and the renal CS. To this end, we extended our previously proposed kidney segmentation framework [24]. Specifically, a coupled formulation of the BEAS framework to simultaneously segment the whole kidney and the renal CS in 3D CT images is used. Using the difference between both surfaces, the renal parenchyma is obtained. Moreover, the gradient-based regularization term proposed in our previous work is now extended to a gradient-based energy functional, which is then combined with a region-based energy.

The rest of this paper is organized as follows. In section II, the general formulation of the coupled BEAS framework and the proposed energy functional for both kidney and renal CS are presented. In section III, the implementation details are outlined, being the results present in section IV. In section 0 , the results are discussed, with the main conclusions being given in section VI.

\section{Methodology}

In this subsection, a description about the proposed methodology to segment the whole kidney, the renal CS, and the renal parenchyma is presented. Firstly, a coupled formulation of the BEAS framework is used to segment he whole kidney and the renal CS simultaneously. Afterwards, the renal parenchyma is segmented by using the different between the kidney and CS segmentations.

\section{A. B-spline Explicit Active Surfaces (BEAS)}

The primary principle of the BEAS framework is the active contours method. In active contours, an evolving interface is propagated by minimizing a given energy functional that reflects the characteristics of the object to be segmented. In BEAS, the concept of Active Geometric Functions (AGF) [26] is used by representing the evolving interface as an explicit function. Geometrically, this implies that one of the coordinates of the points on a surface is given explicitly as a function of the remaining coordinates. Such explicit relation can be mathematically defined as:

$$
\psi: \mathbb{R}^{n-1} \mapsto \mathbb{R}, x_{1}=\psi\left(\boldsymbol{x}^{*}\right),
$$

where $\boldsymbol{x}$ is a point of coordinates $\left\{x_{1}, \ldots, x_{n}\right\}$ within the surface and $\boldsymbol{x}^{*}=\left\{x_{2}, \ldots, x_{n}\right\}$. This explicit form of the interface reduces the dimensionality of the segmentation problem, limiting however the contour's topology. Nevertheless, this formulation fits well to recover the kidney's boundary, owing to its star-like and smooth shape.

The BEAS method extends the concept of AGF to the variational level-set B-spline formulation proposed in [27]. In 
this formulation, the interface function is modeled as a continuous parametric function expressed on a B-spline basis, where the minimization of the energy functional is expressed as a sequence of $1 \mathrm{D}$ convolutions. Therefore, the BEAS method expresses $\psi$ as a linear combination of B-spline basis functions:

$$
x_{1}=\psi\left(x_{2}, \ldots, x_{n}\right)=\sum_{k \in \mathbb{Z}^{n-1}} c[\boldsymbol{k}] \beta^{d}\left(\frac{\boldsymbol{x}^{*}}{h}-\boldsymbol{k}\right),
$$

where $\beta^{d}($.$) is the uniform symmetric (n-1)$-dimensional B-spline of degree $d$. The knots of the B-splines are located on a rectangular grid defined on the chosen coordinate system, with a regular spacing given by $h$. The coefficients of the Bspline representation are gathered in $c[\boldsymbol{k}]$ and the minimization of the energy functional can be directly obtained in terms of these coefficients.

\section{B. Coupled energy functional formulation}

Similarly to typical coupled active contours formulations, we defined both kidney and renal collecting system surfaces separately. In this sense, a simple definition of the boundaries was adapted:

$$
\begin{gathered}
\Gamma_{k}\left(\boldsymbol{x}_{\boldsymbol{k}}^{*}\right)=\psi_{k}\left(\boldsymbol{x}_{\boldsymbol{k}}^{*}\right), \\
\Gamma_{c s}\left(\boldsymbol{x}_{\boldsymbol{c s}}{ }^{*}\right)=\psi_{c s}\left(\boldsymbol{x}_{\boldsymbol{c s}}{ }^{*}\right),
\end{gathered}
$$

for kidney $(k)$ and renal CS interfaces $(C S)$, respectively.

To evolve these interfaces, an appropriate energy criterion must be defined. In [28], Lankton and Tannenbaum introduced the concept of localized region-based energies. Using these energies, the evolving interface is propagated taking into account the properties of the local regions around each point of the interface. The energy functional can be defined in terms of a generic force function $F$ as:

$$
\begin{aligned}
E_{d} & =\int_{\Omega} \delta_{\phi_{k}}\left(\boldsymbol{x}_{\boldsymbol{k}}\right) \int_{\Omega} B\left(\boldsymbol{x}_{\boldsymbol{k}}, \boldsymbol{y}_{\boldsymbol{k}}\right) \cdot F_{k}\left(\boldsymbol{y}_{\boldsymbol{k}}\right) d \boldsymbol{y}_{\boldsymbol{k}} d \boldsymbol{x}_{\boldsymbol{k}} \\
& +\int_{\Omega} \delta_{\phi_{c s}}\left(\boldsymbol{x}_{c s}\right) \int_{\Omega} B\left(\boldsymbol{x}_{c s}, \boldsymbol{y}_{c s}\right) \cdot F_{c s}\left(\boldsymbol{y}_{c s}\right) d \boldsymbol{y}_{c s} d \boldsymbol{x}_{c s},
\end{aligned}
$$

where $\delta_{\phi}(\boldsymbol{x})$ specifies the respective interface and $B(\boldsymbol{x}, \boldsymbol{y})$ is a mask function in which the local parameters are estimated to drive the contour evolution. For each interface, this mask is defined around each point $\boldsymbol{x}$ as the set of points belonging to the normal direction of $\boldsymbol{x}$ and whose distance is lower than a parameter $r$ :

$$
B(\boldsymbol{x}, \boldsymbol{y})= \begin{cases}1, & \text { if } \boldsymbol{y}=\boldsymbol{x}+k \times \widehat{\boldsymbol{N}}, k \in[-r, r] \\ 0, & \text { otherwise }\end{cases}
$$

To minimize the abovementioned energy, a modified version of the gradient descent optimization with feedback step adjustment algorithm is used. In BEAS, the energy can be directly minimized with respect to $c[\boldsymbol{k}]$ through the following evolution equations:

$$
c[\boldsymbol{k}]^{t+1}=c[\boldsymbol{k}]^{t}+\lambda \frac{\partial E}{\partial c[\boldsymbol{k}]^{(t)}}
$$

$$
\begin{aligned}
\frac{\partial E}{\partial c\left[\boldsymbol{k}_{\boldsymbol{i}}\right]}= & \int_{\Gamma_{k}}\left(\bar{g}_{k}\left(\boldsymbol{x}_{\boldsymbol{k}}{ }^{*}\right)\right) \beta^{d}\left(\frac{\boldsymbol{x}_{\boldsymbol{k}}{ }^{*}}{h}-\boldsymbol{k}_{\boldsymbol{i}}\right) d x_{k}{ }^{*} \\
& +\int_{\Gamma_{c s}}\left(\bar{g}_{c s r}\left(\boldsymbol{x}_{c s}{ }^{*}\right)\right) \beta^{d}\left(\frac{\boldsymbol{x}_{c s}{ }^{*}}{h}-\boldsymbol{k}_{\boldsymbol{i}}\right) d x_{c s}{ }^{*},
\end{aligned}
$$

where $t$ is the current iteration number and $\lambda$ is a parameter controlling the step at each iteration. $\bar{g}\left(\boldsymbol{x}^{*}\right)$ is a feature function that reflects the features of the object to be segmented. For clarity's sake, considering a generic function $h(x)$ in $\mathbb{R}^{n}, \bar{h}$ is noted as the restriction of $h$ over the interface $\Gamma$ in $\mathbb{R}^{n-1}$.

\section{Proposed hybrid energy functional}

In this paper, an energy functional that combines regionand edge-based terms is proposed (Figure 2). Concerning the region-based term, a signed version of the Yezzi energy [29] was adopted. This energy proposed by Queirós et al. [30] evolves the contour in order to have the maximum separation between the statistics of the image, modelling the foreground and background regions as having maximally-separated average intensities. To explicitly specify the expected relation between both regions, the signed version of this energy is expressed in terms of $F$ for each surface as:

$$
\begin{gathered}
F_{R_{k}}\left(y_{k}\right)=v_{x_{k}}-u_{x_{k}}, \\
F_{R_{c s}}\left(y_{c s}\right)=u_{x_{c s}}-v_{x_{c s}} .
\end{gathered}
$$

where $u_{x}$ and $v_{x}$ are the mean intensities inside and outside of the respective evolving contour at point $\boldsymbol{x}$. With this energy, the contour related with the kidney surface is forced to evolve so that the interior of the interface has higher intensities than its exterior, assuming that the kidney is brighter than the background (Figure 2C). In opposite, the surface correspondent to the renal CS is evolved assuming that the interior of the kidney (the CS) is darker than the surrounding tissue, i.e. the renal parenchyma (Figure 2E).

The feature functions for the evolution are given by:

$$
\begin{gathered}
\bar{g}_{R_{k}}\left(\boldsymbol{x}_{\boldsymbol{k}}{ }^{*}\right)=\left(\bar{I}\left(\boldsymbol{x}_{\boldsymbol{k}}{ }^{*}\right)-v_{x_{r}}\right)+\left(\bar{I}\left(\boldsymbol{x}_{\boldsymbol{k}}{ }^{*}\right)-u_{x_{r}}\right), \\
\bar{g}_{R_{c s}}\left(\boldsymbol{x}_{c s}{ }^{*}\right)=\left(\bar{I}\left(\boldsymbol{x}_{c s}{ }^{*}\right)-u_{x_{c s}}\right)+\left(\bar{I}\left(\boldsymbol{x}_{c s}{ }^{*}\right)-v_{x_{c s}}\right),
\end{gathered}
$$

where $\bar{I}\left(\boldsymbol{x}^{*}\right)$ corresponds to the image value at point $\boldsymbol{x}$.

In order to make our strategy less dependent of the initialization and the presence of local minima, we combine the abovementioned region-based energy with an edge-base term, taking advantages of the expected well-defined boundaries in CT images. For that, it is proposed to use the derivative of the signal given by the set of points belonging to the mask $B$ to analyze its transitions. The gradient information is used as the localized edge-based energy itself, being expressed as:

$$
\begin{aligned}
& F_{E_{k}}\left(y_{k}\right)=-\nabla \mathrm{P}\left(\boldsymbol{x}_{\boldsymbol{k}}{ }^{*}\right), \\
& F_{E_{c s}}\left(y_{c s}\right)=\nabla \mathrm{P}\left(\boldsymbol{x}_{c s}{ }^{*}\right),
\end{aligned}
$$



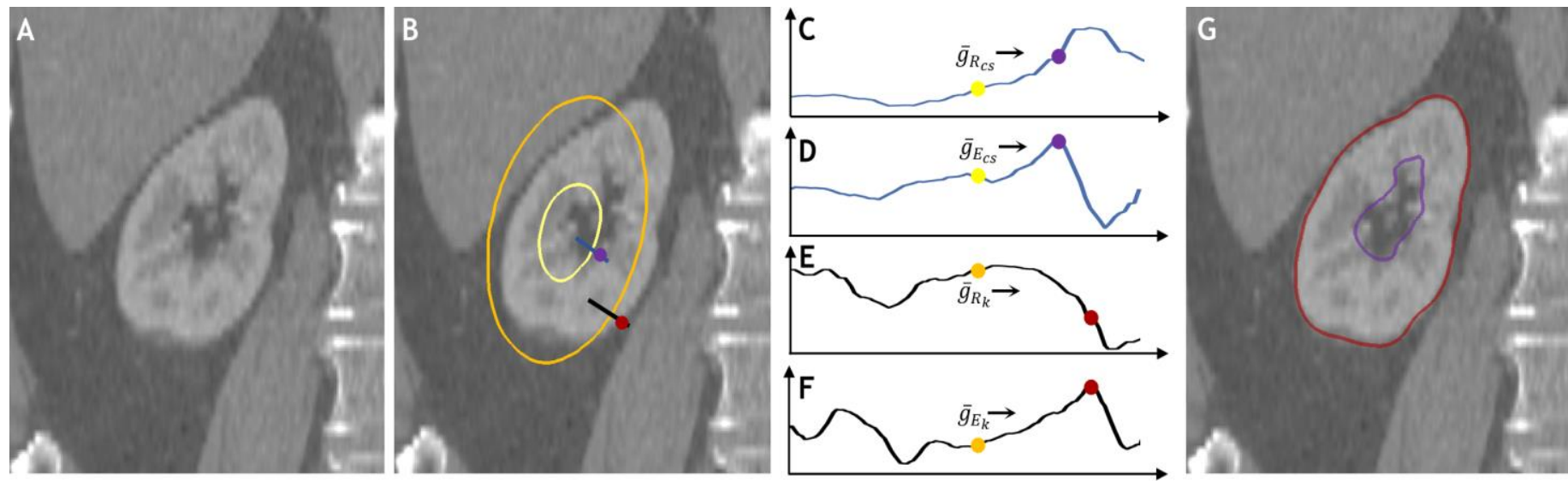

Figure 2 - Principle of the localized energy functional used in the segmentation method. (A) Slice of the original CT image; (B) Contour evolution for kidney (dark yellow) and renal CS (light yellow), showing the kidney's boundary (red dot) and renal CS's boundaries (violet dot); (C) Image intensity profile extracted in the blue line in (B); (D) Derivative profile of (C); (E) Image intensity profile extracted in the black line in (B); (F) Derivative profile of (E); (G) Final segmentation of kidney (red contour) and renal CS (violet contour).

where $\mathrm{P}$ corresponds to the surface's normal profiles computed using mask $B$.

The edge-based term related with kidney's surface presents its maximum in bright-to-dark intensity transitions (Figure 2D), while the edge-based term related with the renal CS surface presents higher values in dark-to-bright steps (Figure $2 \mathrm{~F})$. The feature functions associated to this energy is computed by convolving the energy with the derivative of a Gaussian function $G$ :

$$
\begin{gathered}
\bar{g}_{E_{k}}\left(\boldsymbol{x}_{\boldsymbol{k}}{ }^{*}\right)=\nabla G * F_{E_{k}}(y), \\
\bar{g}_{E_{C S}}\left(\boldsymbol{x}_{c s}{ }^{*}\right)=\nabla G * F_{E_{c s}}(y) .
\end{gathered}
$$

The proposed energy functional consists in the combination of the region-based energy and the proposed edge-based energy, resulting in a hybrid force function $F$, defined in equation (5), expressed as:

$$
\begin{gathered}
F_{k}\left(\boldsymbol{y}_{k}\right)=F_{R_{k}}\left(y_{k}\right)+\alpha_{k} \cdot F_{E_{k}}\left(y_{k}\right) \\
F_{C S}\left(\boldsymbol{y}_{c s}\right)=F_{R_{C S}}\left(y_{c S}\right)+\alpha_{c s} \cdot F_{E_{c S}}\left(y_{c S}\right)
\end{gathered}
$$

where the parameter $\alpha$ is a weight factor that allow to control the influence of edge term in the associated contours evolution. The feature function associated with the proposed energy functional is thus given by:

$$
\begin{gathered}
\bar{g}_{k}\left(\boldsymbol{x}_{\boldsymbol{k}}{ }^{*}\right)=\bar{g}_{R_{k}}\left(\boldsymbol{x}_{\boldsymbol{k}}{ }^{*}\right)+\alpha_{k} \cdot \bar{g}_{E_{k}}\left(\boldsymbol{x}_{\boldsymbol{k}}{ }^{*}\right) \\
\bar{g}_{c s}\left(\boldsymbol{x}_{c s}{ }^{*}\right)=\bar{g}_{R_{c s}}\left(\boldsymbol{x}_{c s}{ }^{*}\right)+\alpha_{c s} \cdot \bar{g}_{E_{c s}}\left(\boldsymbol{x}_{c s}{ }^{*}\right)
\end{gathered}
$$

\section{Regularization term}

In order to guide the surfaces with a proper relation between each other, and to guarantee that the coupling of the surfaces results in plausible segmentations, a regularization term $\bar{g}_{R T}$ is added to the CS energy functional. In [31], a regularization term that ensures a proper local thickness between two surfaces is proposed. Although this term is feasible to constrain the thickness between the kidney and the renal CS, there is a region of the kidney, namely its concave surface (renal hilum), where both kidney and renal CS's surfaces must merge. Here, both surfaces stand in a homogeneous region, presenting similar inner and outer intensities. In this sense, we proposed a new formulation of the regularization term, which allows the CS surface to be evolved towards the kidney's boundary in this homogeneous region:

$$
\begin{aligned}
\bar{g}_{R T}\left(\boldsymbol{x}_{c s}{ }^{*}\right)= & \left(\left(1-H_{h}\right) \cdot\left(\psi_{k}-\psi_{c s}-t_{t}\right)\right) \cdot H_{t} \\
& +H_{h} \cdot\left(\psi_{k}-\psi_{c s}\right)
\end{aligned}
$$

where $H$ is the Heaviside function defined in our implementation as:

$$
\begin{gathered}
H_{h}=H\left(t_{h}-\left|\left(u_{x_{k}}-u_{x_{c s}}\right)-\left(v_{x_{k}}-v_{x_{c S}}\right)\right|\right), \\
H_{t}=H\left(t_{t}-\left(\left|\psi_{k}-\psi_{c s}\right|\right)\right) .
\end{gathered}
$$

In the equation, $t_{t}$ is the minimum thickness between the kidney and CS surfaces in the regions where the surfaces should not merge and $t_{h}$ is a homogeneity parameter. Note that the regularization term is regulated by $t_{h}$, being always activated in homogeneous regions. However, when in heterogenous regions, $t_{t}$ controls the thickness between the surfaces, penalizing thicknesses lower than its value. In this sense, the regularization term ensures a proper thickness between both kidney and CS's surfaces, while pushing the latter one towards the kidney boundary in the renal hilum. The $\mathrm{CS}$ feature function with the regularization term in then given as:

$$
\bar{g}_{c s r}\left(\boldsymbol{x}_{c s}{ }^{*}\right)=\bar{g}_{c s}\left(\boldsymbol{x}_{c s}{ }^{*}\right)+\bar{g}_{R T}\left(\boldsymbol{x}_{c s}{ }^{*}\right) .
$$

\section{EXPERIMENTS}

\section{A. Dataset and ground truth}

The performance of the proposed segmentation method was evaluated on a database of 5 CT images, being both left and right kidneys assessed individually. The parameters used during the acquisition of the CT images were set to $120 \mathrm{kV}$ 
and between 265 and $445 \mathrm{~mA}$. The pixel spacing ranged from 0.61 to $0.96 \mathrm{~mm}$ and the spacing between slices ranged from 1.5 to $3 \mathrm{~mm}$. The number of slices was between 502 and 797 . Each axial slice had a spatial resolution of $512 \times 512$ pixels.

Concerning the ground truth, both kidney and CS's ground truth surfaces were manually delineated by one observer using the Medical Imaging Interaction Toolkit (MITK) software [32]. The ground truth of the renal parenchyma was obtained from the difference of both kidney and CS ground truth segmentations. The manual delineation was performed twice for each structure (henceforward referred as Manual $_{1}$ and Manual $_{2}$ ), allowing the comparison between the performance of the proposed method and the intra-observer variability.

\section{B. Contour initialization}

The first step of active contour-based segmentation strategies is the initialization of the contour that will be evolved towards to the object to be segmented. To initialize the BEAS method in the present work, a user drew fourteen points in the kidney and an ellipsoid was fitted to this data. The fitted ellipsoid was used as initialization for the kidney's surface during semi-automatic segmentation with BEAS. The renal CS surface was initialized within the BEAS framework as follows:

$$
\Gamma_{c s_{0}}=\Gamma_{k_{0}} / 2
$$

\section{Implementation details}

In the implementation of the proposed method, some parameters had to be defined. First, the CT images were normalized between 0 and 1. Next, an appropriate coordinate system was set. Due to the kidney's ellipsoid shape, a spherical coordinate system was chosen. Thus, the radial coordinate of the points within the surfaces will be given as a function of the azimuth and zenith angles. Concerning the Bspline scales and the number of surface points, the first one was set to $2^{2}$ and 64 points were used to define each direction of the rectangular grid in the spherical domain. These values were selected for both kidney and CS's surfaces. Regarding mask $B$, the radius of the normal vector was experimentally set to $10 \mathrm{~mm}$ for kidney and $8 \mathrm{~mm}$ for $\mathrm{CS}$. The weights associated to the region-based and edge-based terms in the hybrid energy functional were set to 1 and 1.75, respectively, for the two surfaces. At last, $t_{t}$ and $t_{h}$ were experimentally set to 5 pixels and 0.1 , respectively.

\section{RESUltS}

TABLE I presents a surface analysis of the segmentation performance against Manual $_{1}$, using Dice, Point-to-Surface (P2S) distance, and 95 ${ }^{\text {th }}$ Hausdorff Distance (HD). In the table, results for the whole kidney, renal $\mathrm{CS}$, and renal parenchyma are presented. The intra-observer variability between both manual analyses is also presented in the table. In Figure 3, an example of one segmentation result is presented.

Besides the surface analysis, a volumetric analysis was also performed. In this analysis, the volumes of the segmented kidney, CS, and parenchyma surfaces were compared with the manual ones. Figure 4 presents the Bland-Altman plots using Manual $_{1}$ as reference. In these analyses, the biases (average difference between methods) and limits of agreement (LOA, $1.96 \sigma$ ) were assessed. The volumetric comparison was also performed between both manual delineations.

The computational time of the proposed strategy was also assessed, being required in average 14 seconds per each

TABLE I.

3D KIDNEY SEGMENTATION PERFORMANCE $(\mu \pm \sigma)$

\begin{tabular}{|c|c|c|c|c|c|c|c|c|c|}
\hline & \multicolumn{3}{|c|}{ DICE $(\%)$} & \multicolumn{3}{|c|}{$\mathrm{P} 2 \mathrm{~S}(\mathrm{~mm})$} & \multicolumn{3}{|c|}{$\mathrm{HD}(\mathrm{mm})$} \\
\hline & Kidney & CS & Parenchyma & Kidney & $\mathrm{CS}$ & Parenchyma & Kidney & $\mathrm{CS}$ & Parenchyma \\
\hline Initialization & $80.9 \pm 3.2$ & $51.2 \pm 12.2$ & - & $4.0 \pm 0.8$ & $5.4 \pm 1.7$ & - & $10.1 \pm 2.0$ & $12.8 \pm 4.0$ & - \\
\hline Segmentation & $92.5 \pm 1.3$ & $63.5 \pm 10.4$ & $86.9 \pm 2.6$ & $1.6 \pm 0.3$ & $4.0 \pm 1.8$ & $1.9 \pm 0.5$ & $5.2 \pm 2.1$ & $11.1 \pm 5.2$ & $6.5 \pm 2.3$ \\
\hline Intra-observer & $94.4 \pm 1.2$ & $71.6 \pm 6.4$ & $91.2 \pm 1.5$ & $1.2 \pm 0.3$ & $2.7 \pm 0.8$ & $1.5 \pm 0.3$ & $3.9 \pm 1.4$ & $6.8 \pm 2.4$ & $4.4 \pm 1.4$ \\
\hline
\end{tabular}
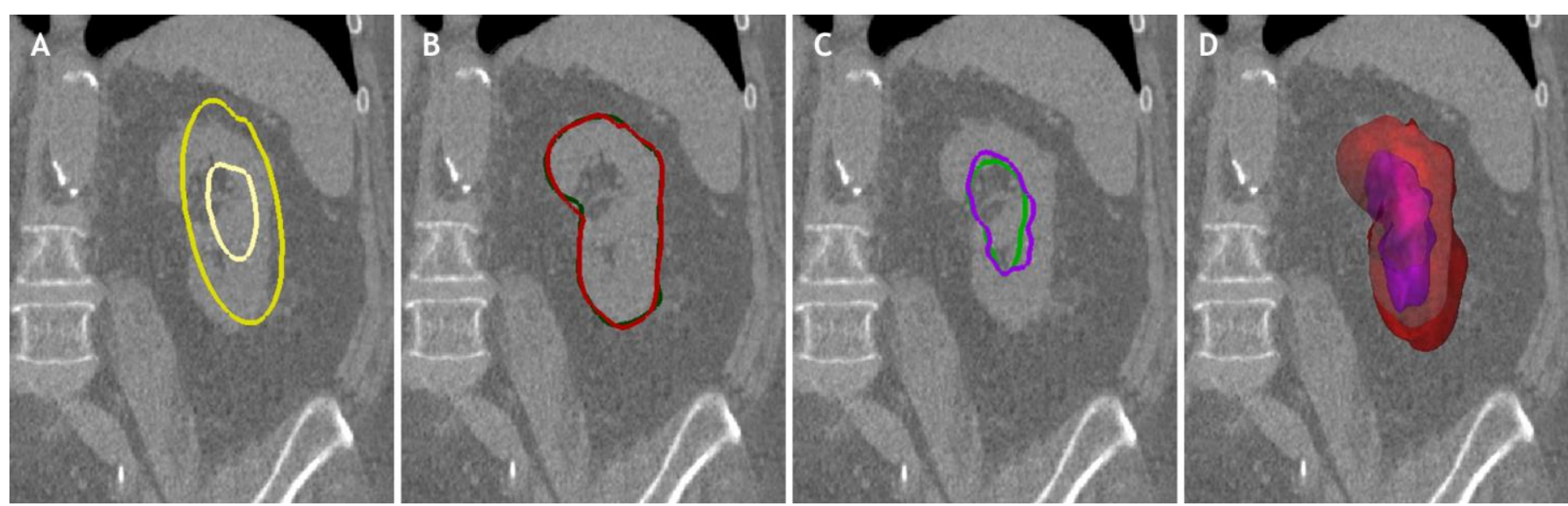

Figure 3- One example segmentation result. (A) Original CT image with initial contours for kidney (dark yellow) and renal CS (light yellow); (B) Final kidney segmentation (red contour) and its respective ground truth (dark green contour); (C) Final renal CS segmentation (violet contour) and its respective ground truth (light green contour); (D) 3D models of the segmentation results; 


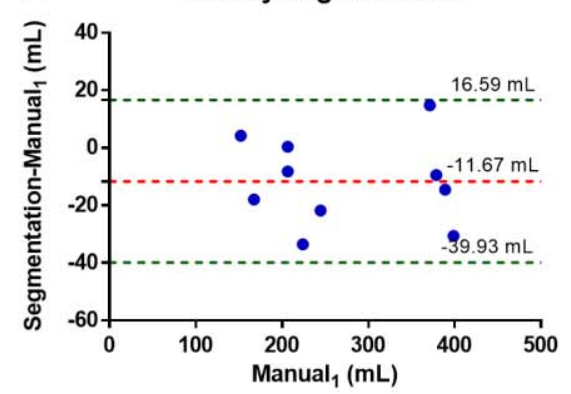

B

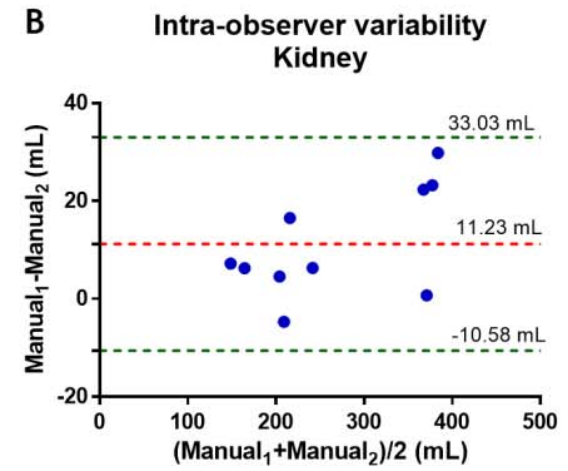

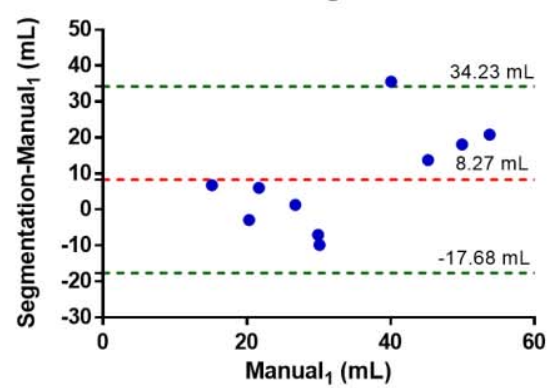

D
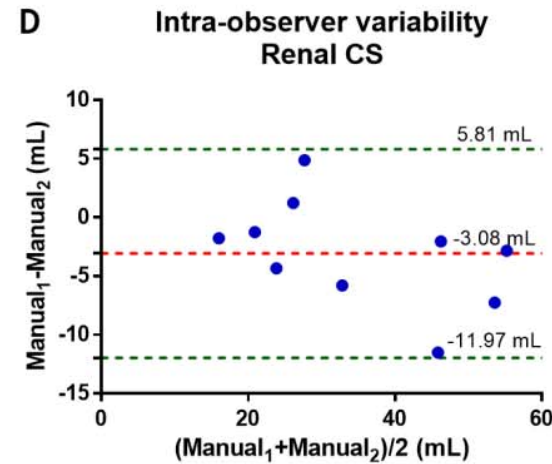

E Renal parenchyma segmentation

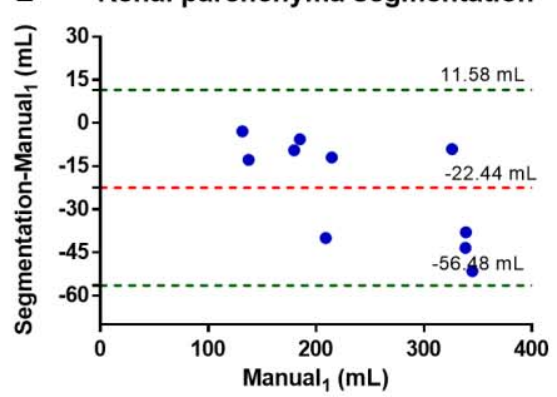

$\mathbf{F}$

Intra-observer variability

Renal parenchyma

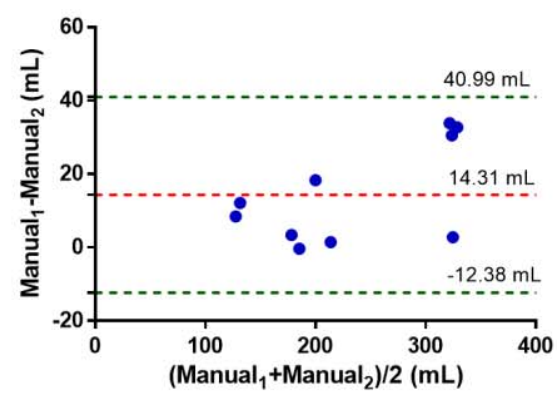

Figure 4- Bland-Altman plots for the volumetric analysis between surfaces obtained by automatic segmentation and manual delineation. (A),(C),(D) represent the agreement between automatic and manual approaches for the different renal structures, while (B),(D),(F) shows the agreement between manual delineations.

segmentation. Note that the results were computed with a MATLAB code running on a $3.6 \mathrm{GHz}$ Core computer with CPU i7-4740 and 16 GB of RAM.

\section{DISCUSSION}

This paper proposed a segmentation approach to extract the kidney, the renal parenchyma, and the renal CS from 3D CT images. Analyzing TABLE I, it is possible to verify the performance of the proposed method. Concerning kidney segmentation, a Dice overlap of $92.5 \%$ and an average P2S error lower than $2 \mathrm{~mm}$ was achieved. These results proved the accuracy of the proposed segmentation method and its associated hybrid energy functional. In [24], we showed that the signed version of the Yezzi energy obtains suitable results for kidney segmentation, which is corroborated in the present paper. When using it, the knowledge of the specific intensity relationship between the kidney and the background adds an explicit prior to the segmentation method, allowing to achieve a good robustness against the different appearances of the kidney. In this sense, this energy was also used to segment the renal CS. However, a purely region-based energy may not be enough if the contour is too far from the true kidney's boundary. To solve this problem, an edge-based term was added to the energy functional, using a gradient-based approach. Therefore, the edge-based term forces the contour to evolve towards positions with higher gradients. Moreover, the edge-based term searches for specific transitions (bright-todark in the case of the kidney segmentation), reducing the influence of interfaces that do not belong to the kidney's real boundaries. In this sense, it is possible to conclude that the combination of both region-based and edge-based terms represents a robust energy functional for kidney segmentation, as demonstrated by the accurate performance of the proposed hybrid energy functional.

In TABLE I, it is also possible to evaluate the accuracy of the proposed method to segment the renal CS. In fact, a Dice overlap of $63.4 \%$ and an average P2S error of $4 \mathrm{~mm}$ were obtained. These results are comparable to the intra-observer agreement, corroborating therefore the potential of this framework. Please note that the renal collecting system has a length of $5 \mathrm{~cm}$ in average. The segmentation of this structure, even manually, is considered a challenging task. First, the renal CS is heterogeneous, given the presence of other substructures with different intensity properties, namely the renal sinus and pelvis, as well as the presence of the point of entrance to the renal vessels. Second, the size and shape variability of the renal $\mathrm{CS}$ among patients can further hamper its segmentation. Another problem that explains the results for the renal CS is the quality of the initialization. As mentioned in section III-B, deformable model approaches are highly dependent of the initialization. In the present paper, the contour initialization for the renal CS relies on an ellipse, similar to the one used for the kidney segmentation, having the same center as the kidney but with a lower radius. However, the renal CS presents a variable size and its position is not completely in the center of the kidney. Instead, it stands nearer to the concave surface of the kidney. Thus, a more robust 
initialization of the renal CS contour can improve the segmentation results.

Last, accurate segmentation results were also achieved for the renal parenchyma, with a Dice overlap of 86.9 and a P2S distance of $1.9 \mathrm{~mm}$. In Figure 3, it is possible to visualize that the proposed segmentation approach resulted in contours close to the ones delineated by the observer, which once again shows the robustness of the proposed method.

When assessing the agreement between manually and semiautomatically computed volumes (Figure 4), no statistically significant biases (red dashed line) were observed for the different structures ( $p>0.05$ in a two-tailed $t$-test against 0 ). Moreover, narrow LOAs (green dashed lines) were obtained for the kidney and the renal parenchyma. In fact, for these structures, the LOAs proved to be statistically similar to the intra-observer ones ( $p>0.05$ in a two-tailed F-Test against the intra-observer), which suggests the equivalence of semiautomatic and manual volume measurements. However, wider LOAs were obtained for the CS structure $(p<0.05)$. This can be explained by segmentations that presented a less accurate result, being this possibly related with bad initializations.

Regarding the computational time, BEAS strategy requires an average of 14 seconds per segmentation. This proves the clear advantages of the BEAS framework for fast 3D segmentation problems. Moreover, the initialization of the contour only requires in average $20 \mathrm{~s}$ for the user input the initial points where the initial ellipsoid is fitted. This proves the advantage of this framework against manual segmentations (which required in average 15 min for delineation).

\section{CONCLUSION}

In this paper, a segmentation method that can be used to provide patient-specific anatomical models of the renal compartments to develop pre-operative patient-specific surgical simulators was proposed. In this sense, the BEAS framework was adapted to a coupled formulation to simultaneously segment the kidney the renal CS. The segmentation of the renal parenchyma was also obtained, being represented by the difference between the other two segmentations. The results obtained showed the potential in terms of accuracy and robustness of the proposed framework for the segmentation of the different renal structures. Moreover, the method proved to be computationally efficient, taking around $14 \mathrm{~s}$ to segment the kidney, parenchyma, and renal CS.

In the future, one intends to develop an automatic kidney detection algorithm to fully automatize the segmentation process, thus obtaining a user-independent methodology. Moreover, a more robust initialization for the renal CS will also be addressed.

\section{ACKNOWLEDGMENTS}

This work was funded by projects NORTE-01-0145FEDER000013, and NORTE-01-0145-FEDER-024300, supported by Northern Portugal Regional Operational Programme (Norte2020), under the Portugal 2020 Partnership Agreement, through the European Regional Development
Fund (FEDER), and also been funded by FEDER funds, through Competitiveness Factors Operational Programme (COMPETE), and by national funds, through the FCTFundação para a Ciência e Tecnologia, under the scope of the project POCI-01-0145-FEDER-007038. The authors acknowledge FCT-Fundação para a Ciência e a Tecnologia, Portugal, and the European Social Found, European Union, for funding support through the Programa Operacional Capital Humano (POCH) in the scope of the $\mathrm{PhD}$ grants SFRH/BD/93443/2013 (S. Queirós) and SFRH/BD/95438/2013 (P. Morais).

\section{REFERENCES}

[1] B.C. Leibovich, M.L. Blute, Surgical Management of Renal Cell Carcinoma, Semin. Oncol. 33 (2006) 552 562. doi:10.1053/j.seminoncol.2006.06.007.

[2] R. Autorino, J.A. Cadeddu, M.M. Desai, M. Gettman, I.S. Gill, L.R. Kavoussi, E. Lima, F. Montorsi, L. Richstone, J.U. Stolzenburg, J.H. Kaouk, Laparoendoscopic single-site and natural orifice transluminal endoscopic surgery in urology: A critical analysis of the literature, Eur. Urol. 59 (2011) 26-45. doi:10.1016/j.eururo.2010.08.030.

[3] A.G. Gallagher, C.U. Cates, Approval of Virtual Reality Training for Carotid Stenting, Jama. 292 (2004) 3024. doi:10.1001/jama.292.24.3024.

[4] R.A. Chaer, B.G. DeRubertis, S.C. Lin, H.L. Bush, J.K. Karwowski, D. Birk, N.J. Morrissey, P.L. Faries, J.F. McKinsey, K.C. Kent, Simulation improves resident performance in catheter-based intervention: Results of a randomized, controlled study, Ann. Surg. 244 (2006) 343-349. doi:10.1097/01.sla.0000234932.88487.75.

[5] F. Nickel, J.A. Brzoska, M. Gondan, H.M. Rangnick, J. Chu, H.G. Kenngott, G.R. Linke, M. Kadmon, L. Fischer, B.P. Müller-Stich, Virtual reality training versus blended learning of laparoscopic cholecystectomy: a randomized controlled trial with laparoscopic novices, Medicine (Baltimore). 94 (2015) e764. doi:10.1097/MD.0000000000000764.

[6] R. Aggarwal, J. Ward, I. Balasundaram, P. Sains, T. Athanasiou, A. Darzi, Proving the effectiveness of virtual reality simulation for training in laparoscopic surgery, Ann. Surg. 246 (2007) 771-779. doi:10.1097/SLA.0b013e3180f61b09.

[7] H. Yamanaka, K. Makiyama, T. Tatenuma, R. Sakata, F. Sano, Y. Kubota, Preparation for pyeloplasty for ureteropelvic junction obstruction using a patientspecific laparoscopic simulator: A case report, J. Med. Case Rep. 6 (2012) 2-5. doi:10.1186/1752-1947-6338.

[8] H.R. Torres, S. Queirós, P. Morais, B. Oliveira, J.C. Fonseca, J.L. Vilaça, Kidney segmentation in ultrasound, magnetic resonance and computed tomography images: A systematic review, Comput. Methods Programs Biomed. 157 (2018) 49-67. doi:10.1016/J.CMPB.2018.01.014. 
[9] M.S. Abirami, T. Sheela, Kidney Segmentation For Finding Its Abnormalities In Abdominal CT Images, Int. J. Appl. Eng. Res. 10 (2015) 32025-32034.

[10] A. Berlgherbi, I. Hadjidj, A. Bessaid, Morphological Segmentation of the Kidneys From Abdominal CT Images, J. Mech. Med. Biol. 14 (2014) 1450073.

[11] E. Zhao, Y. Liang, H. Fan, Contextual informationaided kidney segmentation in CT sequences, Opt. Commun. 290 (2013) 55-62.

[12] C. Jin, F. Shi, D. Xiang, X. Jiang, B. Zhang, X. Wang, W. Zhu, E. Gao, X. Chen, 3D Fast Automatic Segmentation of Kidney Based on Modified AAM and Random Forest, IEEE Trans. Med. Imaging. 35 (2016) 1395-1407.

[13] W. Thong, S. Kadoury, N. Piché, C.J. Pal, W. Thong, S. Kadoury, N. Piché, C.J. Pal, Convolutional networks for kidney segmentation in contrastenhanced CT scans, Comput. Methods Biomech. Biomed. Eng. Imaging Vis. 1163 (2016) 1-6.

[14] J. Liu, M.G. Linguraru, S. Wang, R.M. Summers, Automatic segmentation of kidneys from non-contrast CT images using efficient belief propagation, in: SPIE Med. Imaging. Int. Soc. Opt. Photonics, 2013: pp. 867005-867005-6.

[15] G. Yang, J. Gu, Y. Chen, W. Liu, L. Tang, H. Shu, C. Toumoulin, Automatic Kidney Segmentation in CT Images based on Multi-atlas Image Registration, in: Conf. IEEE Eng. Med. Biol. Soc., 2014: pp. 55385541.

[16] B. Oliveira, S. Queirós, P. Morais, H.R. Torres, J. Gomes-Fonseca, J.C. Fonseca, J.L. Vilaça, A novel multi-atlas strategy with dense deformation field reconstruction for abdominal and thoracic multi-organ segmentation from computed tomography, Med. Image Anal. $45 \quad$ (2018) 108-120. doi:10.1016/J.MEDIA.2018.02.001.

[17] X. Qiao, W. Lu, X. Su, Y.W. Chen, Automatic segmentation method for kidney using dual direction adaptive diffusion flow, in: Innov. Med. Healthc., 2015: pp. 299-307.

[18] R. Cuingnet, R. Prevost, D. Lesage, L.D. Cohen, B. Mory, R. Ardon, Automatic Detection and Segmentation of Kidneys in 3D CT Images Using Random Forests, in: Med. Image Comput. Comput. Interv., 2012: pp. 66-74.

[19] V. Yeghiazaryan, I.D. Voiculescu, Automated 3D renal segmentation based on image partitioning, in: Med. Imaging Image Process., 2016: p. 97842E.

[20] M. Erdt, G. Sakas, Computer aided segmentation of kidneys using locally shape constrained deformable models on CT images, in: SPIE Med. Imaging. Int. Soc. Opt. Photonics, 2010: pp. 762419-762419-8.

[21] F. Khalifa, A. Elnakib, G.M. Beache, G. Gimel'farb, M.A. El-Ghar, R. Ouseph, G. Sokhadze, S. Manning, P. McClure, A. El-Baz, 3D kidney segmentation from CT images using a level set approach guided by a novel stochastic speed function, in: Med. Image
Comput. Comput. Interv., 2011: pp. 587-594.

[22] F. Khalifa, G. Gimel'farb, M. Abo El-Ghar, G. Sokhadze, S. Manning, P. McClure, R. Ouseph, A. ElBaz, A new deformable model-based segmentation approach for accurate extraction of the kidney from abdominal CT images, in: Int. Conf. Image Process., 2011: pp. 3393-3396.

[23] H. Hufnagel, J. Ehrhardt, X. Pennec, A. SchmidtRichberg, H. Handels, Coupled level set segmentation using a point-based statistical shape model relying on correspondence probabilities, in: SPIE Med. Imaging. Int. Soc. Opt. Photonics, 2010: pp. 762318-762318-8.

[24] H.R. Torres, B. Oliveira, S. Queirós, P. Morais, J.C. Fonseca, J. D'hooge, N.F. Rodrigues, J.L. Vilaça, Kidney Segmentation in 3D CT Images Using BSpline Explicit Active Surfaces, in: 4th IEEE Conf. Serious Games Appl. Heal., 2016.

[25] J.E. Pulido, S.L. Furth, S.A. Zderic, D.A. Canning, G.E. Tasian, Renal parenchymal area and risk of ESRD in boys with posterior urethral valves, Clin. J. Am. Soc. Nephrol. 9 (2014) 499-505. doi:10.2215/CJN.08700813.

[26] Q. Duan, E.D. Angelinib, A.F. Lainea, Real-time segmentation by Active Geometric Functions, Comput. Methods Programs Biomed. 98 (2011) 223230. doi:10.1016/j.cmpb.2009.09.001.Real-time.

[27] O. Bernard, D. Friboulet, P. Thévenaz, M. Unser, Variational B-spline level-set: a linear filtering approach for fast deformable model evolution., IEEE Trans. Image Process. 18 (2009) 1179-1191. doi:10.1109/TIP.2009.2017343.

[28] S. Lankton, A. Tannenbaum, Localizing Region-Based Active Contours, IEEE Trans. Image Process. 17 (2008) 2029-2039. doi:10.1109/TIP.2008.2004611.

[29] A. Yezzi, A. Tsai, A. Willsky, A Fully Global Approach to Image Segmentation via Coupled Curve Evolution Equations, J. Vis. Commun. Image Represent. $\quad 13 \quad$ (2002) 195-216. doi:10.1006/jvci.2001.0500.

[30] S. Queirós, D. Barbosa, B. Heyde, P. Morais, J.L. Vilaça, D. Friboulet, O. Bernard, J. D'hooge, Fast automatic myocardial segmentation in 4D cine CMR datasets, Med. Image Anal. 18 (2014) 1115-1131. doi:10.1016/j.media.2014.06.001.

[31] T. Dietenbeck, D. Barbosa, M. Alessandrini, R. Jasaityte, V. Robesyn, J. D'hooge, D. Friboulet, O. Bernard, Whole myocardium tracking in 2Dechocardiography in multiple orientations using a motion constrained level-set, Med. Image Anal. 18 (2014) 500-514. doi:10.1016/j.media.2014.01.005.

[32] I. Wolf, M. Vetter, I. Wegner, M. Nolden, T. Bottger, M. Hastenteufel, M. Schobinger, T. Kunert, H.-P. Meinz, The Medical Imaging Interaction Toolkit (MITK) - a toolkit facilitating the creation of interactive software by extending VTK and ITK, Med. Imaging 2004. (2004) 16-27. 